TRANSACTIONS OF THE

AMERICAN MATHEMATICAL SOCIETY

Volume 360, Number 12, December 2008, Pages 6631-6642

S 0002-9947(08)04512-1

Article electronically published on July 24, 2008

\title{
A FINITE GROUP ACTING ON THE MODULI SPACE OF K3 SURFACES
}

\author{
PAOLO STELLARI
}

\begin{abstract}
We consider the natural action of a finite group on the moduli space of polarized K3 surfaces which induces a duality defined by Mukai for surfaces of this type. We show that the group permutes polarized FourierMukai partners of polarized K3 surfaces and we study the divisors in the fixed loci of the elements of this finite group.
\end{abstract}

\section{INTRODUCTION}

In 11, Mukai introduced a duality for polarized K3 surfaces with Picard number 1. More precisely, given a K3 surface $X$ of this type, Mukai constructs a fine moduli space $Y$ of stable sheaves on $X$ whose Chern classes satisfy some restrictive conditions. Due to [10, $Y$ is also a K3 surface and, by [11, if $X$ has a polarization of degree $2 d$, then $Y$ has a polarization of the same degree. By analogy with the case of abelian varieties, $Y$ is interpreted as a dual variety of $X$. The universal family on $X \times Y$ yields an equivalence of the bounded derived categories of coherent sheaves on $X$ and $Y$. Using the results in [10] it is quite easy to show that the same duality can be defined for K3 surfaces with arbitrary Picard group (Section 2.1).

The aim of this paper is to show that Mukai's duality is given by the natural action of a finite group $G_{2 d}$ on the moduli space $\mathcal{K}_{2 d}$ of K3 surfaces with a polarization of degree $2 d$ ( $d$ is any positive integer). Indeed we prove that $G_{2 d}$ acts by interchanging periods of polarized K3 surfaces with equivalent bounded derived categories of coherent sheaves. Moreover such an action is free and transitive on the set of points of $\mathcal{K}_{2 d}$ parametrizing polarized K3 surfaces with the same derived category and with Picard number 1 . As we will point out, this immediately leads to an interpretation of Mukai's duality as the action of $G_{2 d}$ on $\mathcal{K}_{2 d}$ (see Theorem 2.2 ).

The action of $G_{2 d}$ is not necessarily free on the set of periods of K3 surfaces with Picard number greater than 1. In general, it seems difficult to determine explicitly the irreducible components of the fixed loci and their dimension. Our results on the geometrical properties of the divisors in the fixed loci of the non-trivial elements of $G_{2 d}$ are summarized by Theorem 3.3. It essentially says that the irreducible divisors contained in such fixed loci are Heegner divisors. As a first approximation, a Heegner divisor can be thought of as the image in $\mathcal{K}_{2 d}$ of a hyperplane section of

Received by the editors September 28, 2006 and, in revised form, March 13, 2007.

2000 Mathematics Subject Classification. Primary 14J28, 14J10.

Key words and phrases. K3 surfaces, moduli space of K3 surfaces, Fourier-Mukai partners.

(C)2008 American Mathematical Society

Reverts to public domain 28 years from publication 6631 
the period domain of polarized K3 surfaces, and the K3 surfaces corresponding to its points have Picard number greater than or equal to 2 .

These divisors have recently appeared in other similar contexts. It is the case of 13 where the author studies rank-2 lattices $L$ such that all polarized K3 surfaces $X$ whose Picard group contains $L$ as a primitive sublattice are isomorphic to the moduli space of stable sheaves on $X$ determined by the choice of specific Chern classes (see Remark 3.2 for more details).

The quotient $G_{2 d} \backslash \mathcal{K}_{2 d}$ parametrizes primitive Hodge structures of K3 surfaces with polarizations of degree $2 d$. Such a variety naturally appears when describing divisors of the moduli space of cubic 4-folds. A dense subset of $G_{2 d} \backslash \mathcal{K}_{2 d}$ parametrizes all triangulated categories realized as derived categories of coherent sheaves on K3 surfaces with Picard number 1 (see Section 2.4 for these two remarks). As a consequence of Theorems 2.2 and 3.3. we describe the ramification divisor of the natural map $\mathcal{K}_{2 d} \rightarrow G_{2 d} \backslash \mathcal{K}_{2 d}$ and we show that this dense subset does not meet the singular locus of $G_{2 d} \backslash \mathcal{K}_{2 d}$ (see Corollary 3.4).

\section{Mukai's duality and the action of $G_{2 d}$}

Recall that a lattice is a free abelian group $L$ of finite rank with a non-degenerate symmetric bilinear form $b: L \times L \rightarrow \mathbb{Z}$. We denote by $\mathrm{O}(L)$ the group of isometries of $L$. Given the dual $L^{\vee}:=\operatorname{Hom}(L, \mathbb{Z})$ of a lattice $L$ and the natural inclusion $L \hookrightarrow L^{\vee}$, the discriminant group of $L$ is the quotient $A_{L}:=L^{\vee} / L$. Notice that $A_{L}$ inherits from $L$ a bilinear form with values in $\mathbb{Q} / \mathbb{Z}$. (For more details about lattices and discriminant groups, see 12 .) If $X$ is a $\mathrm{K} 3$ surface, the group $H^{2}(X, \mathbb{Z})$ with the cup product is an even unimodular lattice which is isomorphic to the lattice $\Lambda:=U^{\oplus 3} \oplus E_{8}(-1)^{\oplus 2}$ (for the meaning of $U$ and $E_{8}$, see [2]). Given two K3 surfaces $X$ and $Y$, a Hodge isometry is an isometry $\psi: H^{2}(X, \mathbb{Z}) \rightarrow H^{2}(Y, \mathbb{Z})$ such that $\psi\left(H^{2,0}(X)\right)=H^{2,0}(Y)$. We will denote by $\mathrm{NS}(X), T(X):=\mathrm{NS}(X)^{\perp}$ and $\rho(X):=\operatorname{rkNS}(X)$ respectively the Néron-Severi group, the transcendental lattice and the Picard number of $X$.

The plan of this section is as follows. Mukai's duality is introduced in Section 2.1. The moduli space $\mathcal{K}_{2 d}$ of $2 d$-polarized $\mathrm{K} 3$ surfaces and the action of a finite group $G_{2 d}$ on $\mathcal{K}_{2 d}$ are described in Section 2.2. In that section we also discuss the interpretation of Mukai's duality in terms of the action of $G_{2 d}$. In Section 2.4 we provide a few geometrical interpretations of the quotient $G_{2 d} \backslash \mathcal{K}_{2 d}$, which will be reconsidered in Section 3 ,

2.1. Mukai's duality. There exists a well-known duality for abelian varieties. Indeed, given an abelian variety $A$ one can just consider the abelian variety $\widehat{A}:=$ $\operatorname{Pic}^{0}(A)$. The key aspects of this duality are the following (see [9]):

(A.1) it is an involution, i.e. $\widehat{\widehat{A}}=A$;

(A.2) there exists an equivalence $\mathrm{D}^{\mathrm{b}}(A) \cong \mathrm{D}^{\mathrm{b}}(\widehat{A})$ induced by the Poincaré line bundle on $A \times \widehat{A}$ (for a smooth projective variety $Y, \mathrm{D}^{\mathrm{b}}(Y)$ is the bounded derived category of coherent sheaves on $Y$ );

(A.3) $A$ and $\widehat{A}$ have polarizations of the same degree.

In [11, Mukai proposed an analogous construction for K3 surfaces (i.e. 2-dimensional complex projective smooth varieties with trivial canonical bundle and first Betti number $\left.b_{1}=0\right)$. Recall that, for a positive integer $d$, a polarized K3 surface of degree $2 d$ (or a $2 d$-polarized $K 3$ surface) is a pair $(X, \ell)$, where $X$ is a K3 surface and 
$\ell$ is a primitive vector in the cone $C(X)^{+} \subset \operatorname{Pic}(X) \otimes \mathbb{R}$ spanned by pseudo-ample (i.e. nef and big) divisors of $X$ and $\ell^{2}=2 d$.

Mukai's construction $([10,11)$ is as follows. Suppose that $(X, \ell)$ is a $2 d$-polarized K3 surface with $\ell^{2}=2 d=2 r s$ and $\operatorname{gcd}(r, s)=1$. Consider the fine moduli space $M(r, \ell, s)$ parametrizing $\ell$-stable sheaves $E$ on $X$ such that $\operatorname{rk} E=r, \mathrm{c}_{1}(E)=\ell$ and $\chi(E)=r+s$. Deep results in [10] ensure that $M(r, \ell, s)$ is a K3 surface as well. A K3 surface dual to $X$ is $\widehat{X}:=M(r, \ell, s)$. In complete analogy with the case of abelian varieties, the results in [10, 11] imply that

(B.1) Mukai's duality is an involution, i.e. $\widehat{\widehat{X}}=X$ (see Remark 2.1 (iii) below);

(B.2) there exists an equivalence $\mathrm{D}^{\mathrm{b}}(X) \cong \mathrm{D}^{\mathrm{b}}(\widehat{X})$ induced by the universal family on $X \times \widehat{X}$

(B.3) $\widehat{X}$ has a polarization $\ell^{\prime}$ such that $\left(\ell^{\prime}\right)^{2}=2 d$.

Remark 2.1. (i) Mukai defined this duality in [11] just for K3 surfaces $X$ with Picard number 1 . On the other hand it can be easily extended to polarized K3 surfaces $(X, \ell)$ with no restrictions on $\rho(X)$ using the explicit description in [10] of the lattice and Hodge structures of the second cohomology group of the moduli space $M(r, \ell, s)$, where $r, \ell$ and $s$ satisfy the previous conditions. Indeed, the only delicate part is (B.3). But due to [10], if $v:=(r, \ell, s)$, then there exists a Hodge isometry $\psi: H^{2}(M(r, \ell, s), \mathbb{Z}) \rightarrow v^{\perp} / \mathbb{Z} v$ and $\psi^{-1}((0, \ell, 2 s))$ is a primitive vector in $\mathrm{NS}(M(r, \ell, s))$ with self-intersection $\ell^{2}$.

(ii) Mukai's construction depends very much on the chosen factorization $2 d=$ 2rs. Indeed, [18, Thm. 2.4] shows that there may be many non-isomorphic K3 surfaces dual (in the sense of Mukai) to a K3 surface.

(iii) Condition (B.1) can be better explained as follows: There exists a vector $\widehat{v}:=(\widehat{r}, \widehat{\ell}, \widehat{s}) \in H^{*}(\widehat{X}, \mathbb{Z})$ with $\operatorname{gcd}(\widehat{r}, \widehat{s})=1, \widehat{\ell}$ ample and primitive, $\widehat{\ell}^{2}=\ell^{2}=2 \widehat{r} \widehat{s}$ and such that the fine moduli space $M(\widehat{r}, \widehat{\ell}, \widehat{s})$ of $\widehat{\ell}$-stable sheaves on $\widehat{X}$ (with the specified Chern classes) is isomorphic to $X$.

2.2. The moduli space of $2 d$-polarized K3 surfaces and the action of $G_{2 d}$. Let $h$ be a primitive vector of $\Lambda$ with $h^{2}=2 d$. The orthogonal complement of $h$ in $\Lambda$ is isometric to the lattice

$$
L_{2 d}:=\langle k\rangle \oplus U^{\oplus 2} \oplus E_{8}(-1)^{\oplus 2},
$$

where $k^{2}=-2 d$. The set $D_{2 d}:=\left\{\sigma \in \mathbb{P}\left(L_{2 d} \otimes \mathbb{C}\right): \sigma \cdot \sigma=0\right.$ and $\left.\sigma \cdot \bar{\sigma}>0\right\}$ is an open subset of a quadric in $\mathbb{P}\left(L_{2 d} \otimes \mathbb{C}\right)$. We put $\Gamma(\langle h\rangle):=\{g \in \mathrm{O}(\Lambda): g(h)=h\}$ and $\Gamma_{2 d}:=\operatorname{im}\left(\Gamma(\langle h\rangle) \rightarrow \mathrm{O}\left(L_{2 d}\right)\right)$. It is easy to verify that $\Gamma_{2 d}$ is the kernel of the map $\Psi: \mathrm{O}\left(L_{2 d}\right) \rightarrow \mathrm{O}\left(A_{L_{2 d}}\right)$. The quotient

$$
\mathcal{K}_{2 d}:=D_{2 d} / \Gamma_{2 d}
$$

is an irreducible quasi-projective variety of dimension 19 (see [1, Exposé XIII). By the Torelli Theorem and the surjectivity of the period map, $\mathcal{K}_{2 d}$ is isomorphic to the moduli space of $2 d$-polarized K3 surfaces (see [1, [5]). Notice that by [12, Thm. 1.14.4], the definition of $\mathcal{K}_{2 d}$ is independent of the choice of the primitive vector $h$ with self-intersection $2 d$.

According to Section 2.1] given two integers $r$ and $s$ such that

$$
r, s \text { positive } \quad 2 d=2 r s \quad \operatorname{gcd}(r, s)=1,
$$


Mukai's duality is given by the involution

$$
\delta_{r, s}: \mathcal{K}_{2 d} \longrightarrow \mathcal{K}_{2 d}
$$

sending the period $\left[\sigma_{X}\right]$ parametrizing a $2 d$-polarized $\mathrm{K} 3$ surface $(X, \ell)$ to the pe$\operatorname{riod}\left[\sigma_{r, s}\right]:=\delta_{r, s}\left(\left[\sigma_{X}\right]\right)$ parametrizing the $2 d$-polarized moduli space $\left(M(r, \ell, s), \ell^{\prime}\right)$. Therefore Mukai's duality is realized by the action on $\mathcal{K}_{2 d}$ of the group of automorphisms

$$
\Delta_{2 d}:=\left\langle\delta_{r, s}: r, s \text { satisfying }(\underline{2.1})\right\rangle \subseteq \operatorname{Aut}\left(\mathcal{K}_{2 d}\right)
$$

generated by Mukai's involutions.

Consider the finite group

$$
G_{2 d}:=\mathrm{O}\left(A_{L_{2 d}}\right) /\{ \pm \mathrm{id}\} .
$$

The main result of this section is the following theorem, which will be proved in Section 2.3 and which shows that Mukai's duality can be described in terms of the natural action of $G_{2 d}$.

Theorem 2.2. The group $G_{2 d}$ acts on $\mathcal{K}_{2 d}$ by sending the period of a $2 d$-polarized $K 3$ surface $(X, \ell)$ to a period in the finite set of $2 d$-polarized Fourier-Mukai partners of $(X, \ell)$. Moreover this action is free and transitive on the set of periods corresponding to the Fourier-Mukai partners of any $2 d$-polarized K3 surface with Picard number 1 and $\Delta_{2 d}=G_{2 d}$.

Recall that two K3 surfaces $X_{1}$ and $X_{2}$ are Fourier-Mukai partners if there exists an exact equivalence $\mathrm{D}^{\mathrm{b}}\left(X_{1}\right) \cong \mathrm{D}^{\mathrm{b}}\left(X_{2}\right)$. We write $\operatorname{FM}(X)$ for the set of isomorphism classes of Fourier-Mukai partners of $X$.

Before passing to the proof of Theorem [2.2, we fix some notation which will be used for the rest of this paper. By definition, there exists a (surjective) map $\mathrm{O}\left(L_{2 d}\right) \rightarrow G_{2 d}$. Hence given $f \in \mathrm{O}\left(L_{2 d}\right)$, we write $[f]$ for the image of $f$ in $G_{2 d}$. If $\sigma$ is an element of $D_{2 d}$ and $\pi: D_{2 d} \rightarrow \mathcal{K}_{2 d}$ is the natural projection, $[\sigma]:=\pi(\sigma)$. If $\sigma \in D_{2 d}$, then $T(\sigma)$ is the minimal primitive sublattice of $\Lambda$ such that $\sigma \in T(\sigma) \otimes \mathbb{C}$. We put

$$
\rho([\sigma]):=22-\operatorname{rk} T(\sigma) \quad \text { and } \quad \mathrm{O}_{\sigma}:=\{g \in \mathrm{O}(T(\sigma)): g(\mathbb{C} \sigma)=\mathbb{C} \sigma\} .
$$

Observe that if $[\sigma]=[\tilde{\sigma}]$, then $\mathrm{O}_{\sigma}$ is conjugate to $\mathrm{O}_{\tilde{\sigma}}$. Moreover, we define $\left(X_{\sigma}, \ell_{\sigma}\right)$ to be the $2 d$-polarized $\mathrm{K} 3$ surface corresponding to $[\sigma]$ (in particular $T\left(X_{\sigma}\right) \cong$ $T(\sigma)$ ). The points of $\mathcal{K}_{2 d}$ will often be called periods.

2.3. Proof of Theorem 2.2. Let us start from the following easy lemma.

Lemma 2.3. The group $G_{2 d}$ acts on $\mathcal{K}_{2 d}$ and $G_{2 d} \cong(\mathbb{Z} / 2 \mathbb{Z})^{p(d)-1}$ where $p(1)=1$ and $p(d)$ is the number of distinct primes dividing $d$, if $d \geq 2$.

Proof. Obviously $\mathrm{O}\left(L_{2 d}\right)$ acts on $D_{2 d}$. The subgroup $\Gamma_{2 d}$ is the kernel of the map $\Psi: \mathrm{O}\left(L_{2 d}\right) \rightarrow \mathrm{O}\left(A_{L_{2 d}}\right)$. Hence it is normal in $\mathrm{O}\left(L_{2 d}\right)$ and $\mathrm{O}\left(L_{2 d}\right) / \Gamma_{2 d}$ acts on $\mathcal{K}_{2 d}=\Gamma_{2 d} \backslash D_{2 d}$. Since, under our hypothesis, $\Psi$ is onto (see [12, Thm. 1.14.2]), $\mathrm{O}\left(A_{L_{2 d}}\right) \cong \mathrm{O}\left(L_{2 d}\right) / \Gamma_{2 d}$ acts on $\mathcal{K}_{2 d}$. As -id acts trivially on $D_{2 d}, G_{2 d}$ acts on $\mathcal{K}_{2 d}$. The second part of the statement is [17, Lemma 3.6.1] (see also [14]).

The fact that $G_{2 d}$ acts on $\mathcal{K}_{2 d}$ by sending the period of a $2 d$-polarized K3 surface $(X, \ell)$ to the period of a $2 d$-polarized Fourier-Mukai partner of $(X, \ell)$ is easy. Indeed, given $[\sigma] \in \mathcal{K}_{2 d}$ corresponding to $(X, \ell)$ and an $f \in \mathrm{O}\left(L_{2 d}\right)$, then $f(T(\sigma))=T(f(\sigma))$. If $\left(X^{\prime}, \ell^{\prime}\right)$ corresponds to $[f(\sigma)] \in \mathcal{K}_{2 d}$, then there is a Hodge 
isometry between $T(X)$ and $T\left(X^{\prime}\right)$. To conclude, observe that Orlov proved in 15. (using results of Mukai) that, given two K3 surfaces $X$ and $Y$, the following three conditions are equivalent: (i) $\mathrm{D}^{\mathrm{b}}(X) \cong \mathrm{D}^{\mathrm{b}}(Y)$; (ii) there exists a Hodge isometry $T(X) \cong T(Y)$; (iii) $Y$ is isomorphic to a smooth compact 2-dimensional fine moduli space of stable sheaves on $X$.

Definition 2.4. Let $(X, \ell)$ be a $2 d$-polarized K3 surface. We define the set of isomorphism classes of $2 d$-polarized Fourier-Mukai partners of $(X, \ell)$ :

$$
\mathrm{FM}_{2 d}(X, \ell):=\left\{\left(X^{\prime}, \ell^{\prime}\right): \begin{array}{ll}
(1) & \left(X^{\prime}, \ell^{\prime}\right) \text { is a } 2 d \text {-polarized K3 surface } \\
& (2) \mathrm{D}^{\mathrm{b}}(X) \cong \mathrm{D}^{\mathrm{b}}\left(X^{\prime}\right)
\end{array}\right\} / \cong,
$$

where $\left(X_{1}, \ell_{1}\right) \cong\left(X_{2}, \ell_{2}\right)$ if and only if there exists an isomorphism $\psi: X_{1} \rightarrow X_{2}$ such that $\psi^{*}\left(\ell_{2}\right)=\ell_{1}$.

Lemma 2.5. Let $(X, \ell)$ be a $2 d$-polarized $K 3$ surface. Then the set $\mathrm{FM}_{2 d}(X, \ell)$ is finite.

Proof. By [4, Prop. 5.3], the set $\operatorname{FM}(X)$ is finite. Let $m:=|\operatorname{FM}(X)|$ and let $Y_{1}, \cdots, Y_{m}$ be representatives of the isomorphism classes of the (unpolarized) Fourier-Mukai partners of $X$.

We first show that the number of non-isomorphic $2 d$-polarizations on $Y_{j}$, for $j \in\{1, \ldots, m\}$, is finite. Indeed, consider the set $A:=\left\{c \in C\left(Y_{j}\right)^{+}: c^{2}=2 d\right\}$. Given $c \in A$, by Saint-Donat's result in [16] and Bertini's Theorem, there exists an irreducible $D$ in the linear system $|3 c|$ such that $D^{2}=18 d$. If

$$
B:=\left\{|D|: D \in \operatorname{Div}\left(Y_{j}\right) \text { is irreducible and } D^{2}=18 d\right\},
$$

item (b) of Theorem 0.1 in [19] asserts that $B / \operatorname{Aut}\left(Y_{j}\right)$ is finite. Since there exists an injective map $A \hookrightarrow B$ defined by $c \mapsto|3 c|, Y_{j}$ has only a finite number $P_{d}\left(Y_{j}\right)$ of non-isomorphic $2 d$-polarizations. It is easy to see that

$$
\left|\mathrm{FM}_{2 d}(X, \ell)\right|=\sum_{j=1}^{m} P_{d}\left(Y_{j}\right) .
$$

Hence $\mathrm{FM}_{2 d}(X, \ell)$ is finite.

Consider the dense subset

$$
\mathcal{C}_{\text {gen }} \subseteq \mathcal{K}_{2 d}
$$

parametrizing $2 d$-polarized K3 surfaces with Picard number 1.

Lemma 2.6. If $(X, \ell)$ is a $2 d$-polarized $K 3$ surface corresponding to $[\sigma] \in \mathcal{C}_{\text {gen }}$, then the periods of distinct isomorphism classes in $\mathrm{FM}_{2 d}(X, \ell)$ correspond to distinct points in the orbit $G_{2 d} \cdot[\sigma]$ and $\left|G_{2 d} \cdot[\sigma]\right|=\left|\mathrm{FM}_{2 d}(X, \ell)\right|$.

Proof. Take $(X, \ell)$ and $[\sigma] \in \mathcal{K}_{2 d}$ as in the statement. In particular, $\operatorname{NS}(X) \cong\langle h\rangle$ (here $h$ is a generator of the orthogonal complement of $L_{2 d}$ in $\Lambda$ ). By [12, Thm. 1.14.4], $\langle h\rangle$ and $\operatorname{NS}(X)$ have unique (up to isometries of $\Lambda$ ) primitive embeddings in $\Lambda$. Therefore,

$$
\mathrm{FM}_{2 d}(X, \ell)=\left\{X_{f(\sigma)}: f \in \mathrm{O}\left(L_{2 d}\right)\right\} / \text { isom. }
$$

Given $f \in \mathrm{O}\left(L_{2 d}\right)$, by the Torelli Theorem, $X_{f(\sigma)} \cong X_{\sigma}$ if and only if either $f \in \mathrm{O}_{\sigma}$ or $f$ extends to a Hodge isometry in $\mathrm{O}(\Lambda)$. Let

$$
I_{1}:=\operatorname{im}\left(\mathrm{O}(\langle h\rangle) \rightarrow \mathrm{O}\left(A_{\langle h\rangle}\right) \cong \mathrm{O}\left(A_{L_{2 d}}\right)\right) \quad \text { and } \quad I_{2}:=\operatorname{im}\left(\Psi: \mathrm{O}\left(L_{2 d}\right) \rightarrow \mathrm{O}\left(A_{L_{2 d}}\right)\right) \text {. }
$$


Obviously, $I_{1}=\{ \pm \mathrm{id}\}$ and, by [12, Thm. 1.14.2], $I_{2}=\mathrm{O}\left(A_{L_{2 d}}\right)$. Now [14, Lemma 4.1] implies that the image of the natural composition $\mathrm{O}_{\sigma} \rightarrow \mathrm{O}\left(L_{2 d}\right) \rightarrow \mathrm{O}\left(A_{L_{2 d}}\right)$ is $\{ \pm$ id $\}$.

The results [12, Prop. 1.6.1, Cor. 1.5.2] state that $g \in \mathrm{O}\left(L_{2 d}\right)$ lifts to an isometry in $\mathrm{O}(\Lambda)$ if and only if $\Psi(g) \in\{ \pm \mathrm{id}\}$. In particular, if $g, h \in G_{2 d}$ and $g \neq h$, then $g([\sigma]) \neq h([\sigma])$. Hence $\left\{g([\sigma]): g \in G_{2 d}\right\}$ is the set of periods of the non-isomorphic Fourier-Mukai partners of $X$. Thus we have just shown that distinct isomorphism classes in $\mathrm{FM}_{2 d}(X, \ell)$ correspond to distinct points in the orbit $G_{2 d} \cdot\left[\varphi\left(\sigma_{X}\right)\right]$ and that

$$
\left|G_{2 d} \cdot\left[\varphi\left(\sigma_{X}\right)\right]\right|=\left|\mathrm{FM}_{2 d}(X, \ell)\right| .
$$

(Compare this with the description of $G_{2 d}$ and of its order given in Lemma 2.3])

This concludes the proof of the first part of Theorem 2.2. Now we just need to show that $\Delta_{2 d}=G_{2 d}$.

Let $(X, \ell)$ be a $2 d$-polarized $\mathrm{K} 3$ surface corresponding to $\left[\sigma_{(X, \ell)}\right] \in \mathcal{C}_{\text {gen }}$. Due to [18, Thm. 2.4], for any Fourier-Mukai partner $Y$ of $X$, there are two positive integers $r$ and $s$ satisfying (2.1) and such that $Y$ is isomorphic to the fine moduli space $M(r, \ell, s)$ parametrizing stable sheaves $E$ on $X$ with $\operatorname{rk} E=r, \mathrm{c}_{1}(E)=\ell$ and $\chi(E)-\operatorname{rk} E=s$. Moreover, $M(r, \ell, s)$ has a (unique) $2 d$-polarization $\ell_{X}^{\prime}$. We denote by $\left[\sigma_{\left(M(r, \ell, s), \ell_{X}^{\prime}\right)}\right] \in \mathcal{C}_{\text {gen }}$ the period corresponding to the $2 d$-polarized K3 surface $\left(M(r, \ell, s), \ell_{X}^{\prime}\right)$.

Since $G_{2 d}$ acts by sending the period of a $2 d$-polarized K3 surface $(X, \ell)$ to the period of a $2 d$-polarized Fourier-Mukai partner of $(X, \ell)$, by [10, Thm. 1.4], given $g \in G_{2 d}$ there exist two integers $r$ and $s$ satisfying (2.1) and such that

$$
g:\left[\sigma_{(X, \ell)}\right] \longmapsto\left[\sigma_{\left(M(r, \ell, s), \ell_{X}^{\prime}\right)}\right],
$$

for any $\left[\sigma_{(X, \ell)}\right] \in \mathcal{C}_{\text {gen }}$. In other words,

$$
\left.\delta_{r, s}\right|_{\mathcal{C}_{\text {gen }}}=\left.g\right|_{\mathcal{C}_{\text {gen }}} .
$$

Conversely, given $r$ and $s$ as in (2.1) and $\left[\sigma_{(X, \ell)}\right] \in \mathcal{C}_{\text {gen }}$, by Lemma 2.6 there is a $g \in G_{2 d}$ such that

$$
g\left(\left[\sigma_{(X, \ell)}\right]\right)=\left[\sigma_{\left(M(r, \ell, s), \ell_{X}^{\prime}\right)}\right] .
$$

Reasoning as before, this yields the equality $\left.\delta_{r, s}\right|_{\mathcal{C}_{\text {gen }}}=\left.g\right|_{\mathcal{C}_{\text {gen }}}$.

To conclude the proof of Theorem 2.2, observe that since $\mathcal{C}_{\text {gen }}$ is dense in $\mathcal{K}_{2 d}$, $\delta_{r, s}=g$ whenever $\left.\delta_{r, s}\right|_{\mathcal{C}_{\text {gen }}}=\left.g\right|_{\mathcal{C}_{\text {gen }}}$.

\subsection{A geometrical interpretation. Consider the quotient}

$$
\mathcal{P}_{2 d}:=G_{2 d} \backslash \mathcal{K}_{2 d} .
$$

It is very easy to see that $\mathcal{P}_{2 d}=\mathrm{O}\left(L_{2 d}\right) \backslash D_{2 d}$. Hence, due to the surjectivity of the period map and the Torelli Theorem for K3 surfaces, each point of $\mathcal{P}_{2 d}$ naturally parametrizes all K3 surfaces with Hodge-isometric primitive (with respect to a polarization of degree $2 d$ ) second cohomology groups. Recall that for a $2 d$ polarized K3 surface $(X, \ell)$, its primitive second cohomology group is the orthogonal complement of $\ell$ in $H^{2}(X, \mathbb{Z})$. Such a sublattice naturally inherits form $H^{2}(X, \mathbb{Z})$ a weight-two Hodge structure.

Very often the quotient $\mathcal{P}_{2 d}$ can be seen as a birational model of special divisors of the moduli space $\mathcal{C}$ of cubic 4 -folds (i.e. hypersurfaces of degree 3 in $\mathbb{P}^{5}$ ). This is the case if and only if $2 d$ is not divisible by 4,9 or any odd prime $p \equiv 2(\bmod 3)$. 
Indeed, under these assumptions, one considers, as in [], the divisors $\mathcal{C}_{2 d}$ of $\mathcal{C}$ whose points parametrize cubic 4 -folds $X$ such that the lattice

$$
A(X):=H^{4}(X, \mathbb{Z}) \cap H^{2,2}(X)
$$

contains a primitive positive definite sublattice $K_{2 d}$ with the properties:

- the class $H^{2}$ (here $H$ is the hyperplane section of $X$ ) belongs to $K_{2 d}$;

- $\operatorname{rk} K_{2 d}=2$ and $2 d=\left|A_{K_{2 d}}\right|$.

Interesting examples of divisors of this type are the ones whose generic points parametrize cubic 4 -folds $X$ whose Fano variety $F(X)$ of lines contained in $X$ is birational to the Hilbert scheme $S^{[2]}$, where $S$ is a K3 surface. Now [6. Thm. 1.0.2] allows us to conclude the existence of a birational map $\mathcal{C}_{2 d} \stackrel{\sim}{\rightarrow} \mathcal{P}_{2 d}$.

Let us investigate a little bit more the geometry of $\mathcal{P}_{2 d}$. In particular, consider the dense subset $\mathcal{C}_{\text {gen }} \subset \mathcal{K}_{2 d}$ whose points are the periods of K3 surfaces with Picard number 1. Due to Theorem 2.2 one can consider the dense subset

$$
\widetilde{\mathcal{C}}_{\text {gen }}:=G_{2 d} \backslash \mathcal{C}_{\text {gen }} \subset \mathcal{P}_{2 d} .
$$

Applying once more Theorem 2.2 we see that $\widetilde{\mathcal{C}}_{\text {gen }}$ parametrizes all triangulated categories realized as bounded derived categories of coherent sheaves on K3 surfaces with Picard number 1 and of degree $2 d$.

\section{Fixed loci of the aCtion of $G_{2 d}$ And divisors of $\mathcal{K}_{2 d}$}

By Theorem 2.2. Mukai's duality has no fixed points contained in the dense subset $\mathcal{C}_{\text {gen }}$. On the other hand it may happen that some $[\sigma]$ in the complement of $\mathcal{C}_{\text {gen }}$ is fixed by a non-trivial $\delta \in \Delta_{2 d}$. In this section we study the divisors contained in the fixed loci of non-trivial elements of $\Delta_{2 d}$ or, equivalently, of $G_{2 d}$.

Proceeding in this direction we state a few definitions. Given a primitive sublattice $L \hookrightarrow L_{2 d}$ with signature $(2,18)$, we put

$$
D_{L}:=\left\{\sigma \in \mathbb{P}(L \otimes \mathbb{C}): \sigma^{2}=0 \text { and } \sigma \cdot \bar{\sigma}>0\right\}=\mathbb{P}(L \otimes \mathbb{C}) \cap D_{2 d} .
$$

Such a primitive sublattice defines a divisor $\mathcal{H}_{L}:=\operatorname{im}\left(\pi: D_{L^{\perp}} \longrightarrow \mathcal{K}_{2 d}\right)$, where $\pi$ is the restriction to $D_{L^{\perp}}$ of the natural projection $D_{2 d} \rightarrow \mathcal{K}_{2 d}$. Obviously, if $j \in \Gamma_{2 d}$, then $\mathcal{H}_{L}=\mathcal{H}_{j(L)}$.

Definition 3.1. A divisor $\mathcal{D}$ of $\mathcal{K}_{2 d}$ is a Heegner divisor if there exists a primitive sublattice $L$ of $L_{2 d}$ with signature $(2,18)$ such that $\mathcal{D}=\mathcal{H}_{L}$.

Remark 3.2. Heegner divisors appear also in [13. Indeed Nikulin studies primitive sublattices $L$ of $\Lambda$ such that, given two positive integers $r, s$ and $l \in L\left(l^{2}>0\right)$,

(1) for all polarized K3 surfaces $(X, \ell)$ with a primitive embedding $i: L \hookrightarrow$ $\mathrm{NS}(X)$ and $\ell=i(l)$, there is an isomorphism $X \cong M(r, \ell, s)$;

(2) property (1) does not hold true if we substitute $L$ with a primitive sublattice $L^{\prime} \hookrightarrow L$ with $\operatorname{rk} L^{\prime}<\operatorname{rk} L$ and $l \in L^{\prime}$.

Following Nikulin's terminology, such a lattice $L$ is called a critical polarized $K 3$ Picard lattice. It is clear that a critical polarized K3 Picard lattice of rank 2 defines a Heegner divisor of the moduli space $\mathcal{K}_{l^{2}}$ of $l^{2}$-polarized K3 surfaces. Moreover, the generic points of these divisors (i.e. the periods in $\mathcal{K}_{l^{2}}$ parametrizing K3 surfaces $X$ with $\operatorname{NS}(X) \cong L$ ) are fixed points of Mukai's duality if $\operatorname{gcd}(r, s)=1$ (simply use Theorem 2.2). 
For a Heegner divisor $\mathcal{H}_{L}$ we denote by $V\left(\mathcal{H}_{L}\right)$ the orthogonal complement of $L$ in $L_{2 d}$. If $\beta \in L_{2 d}$, we write

$$
\beta=\alpha_{\beta} k+m_{\beta} j_{\beta} \in L_{2 d}=\langle k\rangle \oplus U^{\oplus 2} \oplus E_{8}(-1)^{\oplus 2},
$$

where $j_{\beta} \in U^{\oplus 2} \oplus E_{8}(-1)^{\oplus 2}$ is primitive and $\alpha_{\beta}, m_{\beta} \in \mathbb{Z}$. Consider the set

$$
\Re:=\left\{\langle\beta\rangle \subset L_{2 d}: \begin{array}{l}
\text { (a) } \beta \in L_{2 d} \text { is primitive and } \beta^{2} \text { divides } 2 m_{\beta} \text { and } 2 d \\
\text { (b) } \beta^{2}<0, \beta^{2} \neq-2 \text { and } \beta^{2} \neq-2 d
\end{array}\right\} .
$$

In Sections 3.1 and 3.2 we will prove the following result:

Theorem 3.3. The irreducible divisors of $\mathcal{K}_{2 d}$ contained in the union of the fixed loci of the non-trivial elements of $G_{2 d}$ are the Heegner divisors $\mathcal{H}_{L}$ such that $V\left(\mathcal{H}_{L}\right) \in \Re$.

As a consequence of Theorems 2.2 and 3.3 we can prove the following:

Corollary 3.4. The irreducible components of the ramification divisor of the natural map

$$
p: \mathcal{K}_{2 d} \longrightarrow \mathcal{P}_{2 d}
$$

are the Heegner divisors $\mathcal{H}_{L}$ such that $V\left(\mathcal{H}_{L}\right) \in \Re$. Moreover $\widetilde{\mathcal{C}}_{\text {gen }}$ is contained in the smooth locus of $\mathcal{P}_{2 d}$.

Proof. The first part is just Theorem 3.3. For the second part observe that, due to Theorem 2.2, $\operatorname{Sing}\left(\mathcal{P}_{2 d}\right) \cap \widetilde{\mathcal{C}}_{\text {gen }}=\operatorname{Sing}\left(\mathcal{K}_{2 d}\right) \cap \mathcal{C}_{\text {gen }}$, where $\operatorname{Sing}\left(\mathcal{P}_{2 d}\right)$ and $\operatorname{Sing}\left(\mathcal{K}_{2 d}\right)$ are the singular loci of $\mathcal{P}_{2 d}$ and $\mathcal{K}_{2 d}$ respectively. But given $[\sigma] \in \mathcal{K}_{2 d}$ corresponding to a $2 d$-polarized K3 surface $\left(X_{\sigma}, \ell_{\sigma}\right)$ with $\rho\left(X_{\sigma}\right)=1, \mathrm{O}_{\sigma}=\{ \pm$ id $\}$ (see [14, Lemma $4.1])$. Hence $[\sigma] \notin \operatorname{Sing}\left(\mathcal{K}_{2 d}\right)$ and this concludes the proof.

3.1. Fixed loci and reflections. As a first step towards the proof of Theorem 3.3. we show that a divisor in the fixed locus of a non-trivial $g \in G_{2 d}$ is a union of Heegner divisors. Moreover, if $g \in G_{2 d}$ is non-trivial and fixes a divisor, then it is representable by a reflection.

Definition 3.5. (i) If $g \in G_{2 d}$, the set $\operatorname{Fix}(g):=\left\{[\sigma] \in \mathcal{K}_{2 d}: g([\sigma])=[\sigma]\right\}$ is the fixed locus of $g$.

(ii) Let $\beta \in L_{2 d}$ be such that $\beta^{2} \neq 0$ and $\frac{2 x \cdot \beta}{\beta^{2}} \beta \in L_{2 d}$, for every $x \in L_{2 d}$. Then the isometry $r_{\beta} \in \mathrm{O}\left(L_{2 d}\right)$ defined by

$$
r_{\beta}(x):=x-2 \frac{x \cdot \beta}{\beta^{2}} \beta,
$$

for any $x \in L_{2 d}$, is the reflection with respect to $\beta$.

(iii) The subset of $G_{2 d}$ containing the non-trivial elements representable by reflections with respect to vectors with negative self-intersection is denoted by

$$
\operatorname{Ref}\left(G_{2 d}\right):=\left\{g \in G_{2 d} \backslash\{\operatorname{id}\}: g=\left[r_{\beta}\right] \text {, with } \beta^{2}<0 \text { and } \beta \in L_{2 d}\right\} .
$$

Observe that, due to Theorem 2.2 , if $[\sigma] \in \mathcal{K}_{2 d}$ is fixed by some non-trivial $g \in G_{2 d}$, then $\rho([\sigma]) \geq 2$. Under this additional requirement, we can prove the following:

Lemma 3.6. A point $[\sigma] \in \mathcal{K}_{2 d}$ with $\rho([\sigma])=2$ is fixed by at most one non-trivial $g \in G_{2 d}$. 
Proof. If $\left|G_{2 d}\right|=1$ the result is trivial. So, we suppose $\left|G_{2 d}\right|>1$.

It is very easy to observe that if $[\sigma] \in \mathcal{K}_{2 d}$, then $[\sigma] \in \operatorname{Fix}(g)$ if and only if there exists an $f \in \mathrm{O}\left(L_{2 d}\right)$ such that $[f]=g, f(T(\sigma)) \subseteq T(\sigma)$ and $\left.f\right|_{T(\sigma)} \in \mathrm{O}_{\sigma}$. Thus the proposition is proved if, given

$$
S_{\sigma}:=\left\{f \in \mathrm{O}\left(L_{2 d}\right): \begin{array}{ll}
\text { (a) } f(T(\sigma)) \subseteq T(\sigma) & \text { (b) }\left.f\right|_{T(\sigma)} \in \mathrm{O}_{\sigma}
\end{array}\right\}
$$

we show that $\left|\Xi\left(S_{\sigma}\right)\right| \leq 2$, where $\Xi$ is the composition of $\Psi: \mathrm{O}\left(L_{2 d}\right) \rightarrow \mathrm{O}\left(A_{L_{2 d}}\right)$ with the natural projection from $\mathrm{O}\left(A_{L_{2 d}}\right)$ onto $G_{2 d}$.

As $\rho([\sigma])=2$, there is a primitive $\beta \in L_{2 d}$ (unique up to sign) such that $\left(L_{2 d}\right)_{\mathbb{Q}}=$ $\langle\beta\rangle_{\mathbb{Q}} \oplus T(\sigma)_{\mathbb{Q}}$, where, for a lattice $L$, we write $L_{\mathbb{Q}}:=L \otimes_{\mathbb{Z}} \mathbb{Q}$. This decomposition of $\left(L_{2 d}\right)_{\mathbb{Q}}$ induces an injective homomorphism

$$
S_{\sigma} \hookrightarrow \mathrm{O}(\langle\beta\rangle) \times \mathrm{O}(T(\sigma)) .
$$

From Proposition B.1 in 7 it follows that $\operatorname{im}\left(S_{\sigma} \rightarrow \mathrm{O}(T(\sigma))\right)$ is a cyclic group of order $2 n$. Moreover, $\mathrm{O}(\langle\beta\rangle)=\{ \pm \mathrm{id}\}$. Hence $S_{\sigma}$ can be generated by at most two elements and so $\left|\Psi\left(S_{\sigma}\right)\right| \leq 4$ (indeed, due to Lemma 2.3, for any $g \in G_{2 d}, g^{2}=$ id). Since $-\mathrm{id}_{L_{2 d}} \in \mathrm{O}\left(L_{2 d}\right)$ lies in $S_{\sigma}$ and it maps to $(-\mathrm{id},-\mathrm{id})$ in $\mathrm{O}(\langle\beta\rangle) \times \mathrm{O}(T(\sigma))$, $\Xi\left(S_{\sigma}\right)$ can be generated by one element and thus $\left|\Xi\left(S_{\sigma}\right)\right| \leq 2$.

Remark 3.7. Assume that $[\sigma] \in \mathcal{K}_{2 d}$ is a zero-dimensional irreducible component of $\operatorname{Fix}(g)$, for some non-trivial $g \in G_{2 d}$. Then $\rho([\sigma])$ is even. Indeed, generalizing the proof of [14, Lemma 4.1], we see that for each period $[\sigma]$ corresponding to a K3 surface with odd Picard number, $\mathrm{O}_{\sigma}=\{ \pm \mathrm{id}\}$. Thus if $\rho([\sigma])$ is odd and $[\sigma] \in \operatorname{Fix}(g)$, then $\mathcal{H}_{T(\sigma)}:=\operatorname{im}\left(D_{T(\sigma)} \rightarrow \mathcal{K}_{2 d}\right)$ is contained in $\operatorname{Fix}(g)$ and it is a subvariety of codimension $0<\rho([\sigma])-1<19$ in $\mathcal{K}_{2 d}$. As $[\sigma] \in \mathcal{H}_{T(\sigma)}$, it follows that $[\sigma]$ is not a zero-dimensional irreducible component of $\operatorname{Fix}(g)$.

Now we consider the irreducible divisors of $\mathcal{K}_{2 d}$ contained in the fixed loci.

Proposition 3.8. A divisor $\mathcal{D}$ of $\mathcal{K}_{2 d}$ is fixed by at most one non-trivial $g \in G_{2 d}$. In this case $g \in \operatorname{Ref}\left(G_{2 d}\right)$ and the irreducible components of $\mathcal{D}$ are Heegner divisors. Conversely, if $g \in \operatorname{Ref}\left(G_{2 d}\right)$, then $\operatorname{Fix}(g)$ contains a Heegner divisor.

Proof. Obviously, $[\sigma] \in \mathcal{D} \subseteq \operatorname{Fix}(g)$ for a non-trivial $g \in G_{2 d}$ if and only if there exists an $f \in \mathrm{O}\left(L_{2 d}\right)$ such that $[f]=g$ and $\sigma$ is an eigenvector of the $\mathbb{C}$-linear extension $f_{\mathbb{C}}$ of $f$ for a certain complex eigenvalue $\lambda$. More precisely, $\lambda= \pm 1$; otherwise the eigenspace of $\lambda$ would have dimension less than or equal to 10 (indeed, $\bar{\lambda}$ is also an eigenvalue of $f_{\mathbb{C}}$ ).

This and the fact that, due to Theorem 2.2, a point $[\sigma]$ such that $\rho([\sigma])=1$ is not fixed, imply that there exist a subset $S \subseteq\left\{f \in \mathrm{O}\left(L_{2 d}\right):[f]=g\right\}$ and, for every $f \in S$, a non-trivial $\beta_{f} \in L_{2 d}$ such that either $\left.f_{\mathbb{C}}\right|_{\beta_{f}^{\perp}}=$ id or $\left.f_{\mathbb{C}}\right|_{\beta_{f}^{\perp}}=-$ id and

$$
\mathcal{D}=\pi\left(\bigcup_{f \in S} \mathbb{P}\left(\beta_{f}^{\perp} \otimes \mathbb{C}\right) \cap D_{2 d}\right),
$$

where $\pi: D_{2 d} \rightarrow \mathcal{K}_{2 d}$ is the usual projection. This means that $\mathcal{D}$ is the union of the Heegner divisors $\left\{\mathcal{H}_{\beta_{f}^{\perp}}\right\}_{f \in S}$.

A Heegner divisor $\mathcal{H}_{L}$ of $\mathcal{K}_{2 d}$, where $L$ is a primitive sublattice of $L_{2 d}$ whose signature is $(2,18)$, is irreducible. Indeed, by [8, Cor. 2.5], there is a primitive embedding $M:=L^{\perp} \hookrightarrow U \oplus U$ and hence a primitive embedding $U \hookrightarrow M^{\perp}$. 
Take $D_{M^{\perp}}$ and the groups $\Gamma(M):=\{g \in \mathrm{O}(\Lambda): g(m)=m$ for any $m \in M\}$ and $\Gamma_{M}:=\operatorname{im}\left(\Gamma(M) \rightarrow \mathrm{O}\left(M^{\perp}\right)\right)$. By [5, Prop. 5.6], the quotient $\mathcal{K}_{M}:=D_{M^{\perp}} / \Gamma_{M}$ is irreducible. Since $\Gamma(M) \subseteq \Gamma(\langle h\rangle)$, there exists a surjective map $\mathcal{K}_{M} \rightarrow \mathcal{H}_{L}$ and thus $\mathcal{H}_{L}$ is irreducible. From this we conclude that the irreducible components of $\mathcal{D}$ are Heegner divisors.

A straightforward consequence of the previous remarks and of (3.2) is that, when $f \in S, f=r_{\beta_{f}}$ because $\left.f\right|_{\beta_{f}^{\perp}}$ is \pm id. Thus if a Heegner divisor $\mathcal{H}_{L}$ is contained in $\operatorname{Fix}(g)$, for $g \in G_{2 d}$ non-trivial, then $g \in \operatorname{Ref}\left(G_{2 d}\right)$. Now let us suppose that $\mathcal{H}_{L} \subset \operatorname{Fix}\left(g_{1}\right) \cap \operatorname{Fix}\left(g_{2}\right)$, for $g_{1}, g_{2} \in G_{2 d}$ non-trivial. Lemma 3.6, applied to $[\sigma] \in \mathcal{H}_{L}$ with $\rho([\sigma])=2$, shows that $g_{1}=g_{2}$. Hence, any divisor $\mathcal{D}$ is fixed by at most one non-trivial $g \in G_{2 d}$.

The last statement of the proposition is trivial.

3.2. Non-empty fixed loci. In this section we conclude the proof of Theorem 3.3. describing the Heegner divisors fixed by the non-trivial elements of $G_{2 d}$.

Lemma 3.9. If $L$ is a primitive sublattice of $L_{2 d}$ with signature $(2,18)$, then the Heegner divisor $\mathcal{H}_{L}$ is fixed by a non-trivial $g \in G_{2 d}$ if and only if $V\left(\mathcal{H}_{L}\right) \in \Re$.

Proof. A Heegner divisor $\mathcal{H}_{L}$ with $V\left(\mathcal{H}_{L}\right)=\langle\beta\rangle$, for some primitive $\beta \in L_{2 d}$ is fixed by a non-trivial $g \in G_{2 d}$ if and only if $\beta$ defines a reflection $r_{\beta}$ such that $\left[r_{\beta}\right]=g \in \operatorname{Ref}\left(G_{2 d}\right)$ (see Proposition 3.8).

Suppose that $\beta$ defines a reflection in $\mathrm{O}\left(L_{2 d}\right)$ which is non-trivial in $G_{2 d}$. As at the beginning of Section 3, let us write $\beta=\alpha k+m j \in\langle k\rangle \oplus U^{\oplus 2} \oplus E_{8}(-1)^{\oplus 2}$, where $j \in U^{\oplus 2} \oplus E_{8}(-1)^{\oplus 2}$ is primitive and $\alpha, m \in \mathbb{Z}$. Let $-\beta^{2} / 2=p^{e} b_{1}$, with $\left(b_{1}, p\right)=1$ and $p$ prime. Since $j \in U^{\oplus 2} \oplus E_{8}(-1)^{\oplus 2}$ and $j \neq 0$ (otherwise $\left[r_{\beta}\right]=$ id $\left.\in G_{2 d}\right)$ there exists an $x \in L_{2 d}$ such that $x \cdot \beta=m$. Since $\frac{2(x \cdot \beta)}{\beta^{2}} \beta \in L_{2 d}$, for all $x \in L_{2 d}$ and $\beta$ is primitive, $p^{e}$ divides $m$ but not $\alpha$. Moreover $p^{e}$ divides $d$, because $\beta^{2}=-2 d \alpha^{2}+m^{2} j^{2}$. Thus $\beta$ satisfies (a) in (3.1).

Notice that a reflection $r_{\beta} \in \mathrm{O}\left(L_{2 d}\right)$ with $\beta^{2}<0$ is such that $\left[r_{\beta}\right] \in \operatorname{Ref}\left(G_{2 d}\right)$ if and only if (A) $\frac{2 d}{\beta^{2}} \alpha^{2} \not \equiv 0(\bmod d)$ and $(\mathrm{B}) 1+\frac{2 d}{\beta^{2}} \alpha^{2} \not \equiv 0(\bmod d)$. Indeed, by the very definitions of the discriminant group $A_{L_{2 d}}$ and of the natural map $\mathrm{O}\left(L_{2 d}\right) \rightarrow \mathrm{O}\left(A_{L_{2 d}}\right), r_{\beta}$ is trivial in $G_{2 d}$ if and only if either $r_{\beta}(k)=\left(2 d m_{1}+1\right) k+l_{1}$ or $r_{\beta}(k)=\left(2 d m_{2}-1\right) k+l_{2}$, for $m_{1}, m_{2} \in \mathbb{Z}$ and $l_{1}, l_{2} \in U^{\oplus 2} \oplus E_{8}(-1)^{\oplus 2} \hookrightarrow L_{2 d}$. This gives exactly (A) and (B).

Since $\beta^{2} / 2$ does not divide $\alpha^{2}$, it follows that (A) and (B) hold true if and only if (b) in (3.1) holds. Hence $V\left(\mathcal{H}_{L}\right)=\langle\beta\rangle \in \Re$ and this proves the "if" direction. The converse is easy and left to the reader.

We discuss now two examples which show that the fixed loci of the non-trivial elements of $G_{2 d}$ may or may not contain a Heegner divisor.

Example 3.10. Suppose $2 d=4 p$, where $p$ is an odd prime such that $p$ is not a square modulo 4 and 2 is not a square modulo $p$. By Lemma 2.3, $G_{2 d}=\langle g\rangle \cong \mathbb{Z} / 2 \mathbb{Z}$. Let $\beta_{1}:=\alpha_{1} k+2 j_{1}=(1,2, p-1,0, \ldots, 0) \in L_{2 d}=\langle k\rangle \oplus U^{\oplus 2} \oplus E_{8}(-1)^{\oplus 2}$. As $\left\langle\beta_{1}\right\rangle \in \Re$, by Lemma 3.9. $\mathcal{H}_{\beta_{1}^{\perp}} \subseteq \operatorname{Fix}(g)$ and $\beta_{1}^{2}=-4$. Moreover Fix $(g)$ does not contain any other Heegner divisor. Indeed, suppose that there exists a primitive $\beta_{2} \in L_{2 d}$ such that $\mathcal{H}_{\beta_{2}^{\perp}} \subseteq \operatorname{Fix}(g)$. Using Lemma 3.9, we have $\left\langle\beta_{2}\right\rangle \in \Re$ and $\beta_{2}^{2}=-4$.

Let $\beta_{2}=\alpha_{2} k+2 j_{2}$, where $j_{2} \in U^{\oplus 2} \oplus E_{8}(-1)^{\oplus 2}$ ( $j_{2}$ is not necessarily primitive). Since $-4=\beta_{2}^{2}=-2 d \alpha_{2}^{2}+4 j_{2}^{2}, \alpha_{2}^{2} \equiv 1(\bmod 2)$ and there exists an integer $w$ 
such that $\alpha_{2}=1+2 w$. Observe that $\frac{1}{2} j_{2} \notin U^{\oplus 2} \oplus E_{8}(-1)^{\oplus 2}$. Indeed, if $\frac{1}{2} j_{2} \in$ $U^{\oplus 2} \oplus E_{8}(-1)^{\oplus 2}$, then there would exist an integer $\alpha$ such that $p \alpha^{2} \equiv 1(\bmod 4)$. This is impossible since $p$ is not a square modulo 4 .

Let $L_{i}$ be the minimal primitive sublattice of $\Lambda$ containing the lattice generated by $h$ and $\beta_{i}$. An integral basis of $L_{i}$ is given by $e_{i}:=h$ and $f_{i}:=\frac{2}{\beta^{2}}\left(\alpha_{i} h-\beta_{i}\right)$. We can now define an isometry $\psi: L_{2} \rightarrow L_{1}$ such that $e_{2} \mapsto e_{1}$ and $f_{2} \mapsto-w e_{1}+f_{1}$. Due to [12, Thm. 1.14.4], $\psi$ extends to an isometry $\psi^{\prime} \in \mathrm{O}(\Lambda)$. Hence $\mathcal{H}_{\beta_{1}^{\perp}}=\mathcal{H}_{\beta_{\frac{2}{2}}}$.

As a very special case, we have Example 1.3 in [1] where Mukai's duality is studied for K3 surfaces with Picard number 1 and with a polarization of degree 12 . It is quite easy to see that, if $\mathcal{H}_{L}$ is the unique Heegner divisor in the fixed locus of the non-trivial $g \in G_{2 d}$ and $[\sigma] \in \mathcal{H}_{L}$ is such that $\rho([\sigma])=2$, then

$$
\mathrm{NS}\left(X_{\sigma}\right) \cong\left(\mathbb{Z}^{2},\left(\begin{array}{ll}
2 & 4 \\
4 & 2
\end{array}\right)\right)
$$

In particular $\operatorname{NS}\left(X_{\sigma}\right)$ is isometric to the Néron-Severi group of a generic K3 surface which is a complete intersection of bi-degree $(1,1)$ and $(2,2)$ in $\mathbb{P}^{2} \times \mathbb{P}^{2}$. These surfaces were studied by Wehler in [20].

Example 3.11. There are $2 d$-polarizations such that none of the non-trivial elements of $G_{2 d}$ fixes a Heegner divisor. An example is when $d=15$. Indeed, in this case, $G_{2 d}=\{\mathrm{id}, g\}$ (see Lemma 2.3) and if there exists a Heegner divisor $\mathcal{H}_{L} \subseteq \operatorname{Fix}(g)$ with $V\left(\mathcal{H}_{L}\right)=\langle\beta\rangle$, then, by Lemma 3.9, $\beta^{2}$ is either -6 or -10 . If $\beta^{2}=-6$, then $-15 \alpha^{2}+3^{2} s=-d \alpha^{2}+m^{2} \frac{j^{2}}{2}=\frac{\beta^{2}}{2}=-3$ for some integer $s$. In particular, 5 should be a square modulo 3 but this is not the case. Similarly, $\beta^{2}$ cannot be equal to -10 . Hence $\operatorname{Fix}(g)$ does not contain a divisor.

\section{ACKNOWLEDGEMENTS}

During the preparation of this paper the author was partially supported by the MIUR of the Italian Government in the framework of the National Research Project "Geometry on Algebraic Varieties" (Cofin 2004). It is a pleasure to thank Bert van Geemen for useful discussions and many suggestions about the results in this paper. The author thanks Gilberto Bini for comments on earlier versions of the paper.

\section{REFERENCES}

[1] Géométrie des surfaces K3: modules et périodes, Astérisque 126 (1985). MR0785216 (87h:32052)

[2] W. Barth, K. Hulek, C. Peters, A. Van de Ven, Compact complex surfaces, Springer-Verlag, Berlin (2004). MR 2030225 (2004m:14070)

[3] C. Borcea, K3 surfaces and complex multiplication, Rev. Roumaine Math. Pures Appl. 31 (1986), 499-505. MR856201 (88f:11059)

[4] T. Bridgeland, A. Maciocia, Complex surfaces with equivalent derived categories, Math. Z. 236 (2001), 677-697. MR:1827500 (2002a:14017)

[5] I.V. Dolgachev, Mirror symmetry for lattice polarized K3 surfaces, J. Math. Sci. 81 (1996), 2599-2630. MR1420220 (97i:14024)

[6] B. Hassett, Special cubic fourfolds, Compositio Math. 120 (2000), 1-23. MR1738215 (2001g:14066)

[7] S. Hosono, B.H. Lian, K. Oguiso, S.-T. Yau, Fourier-Mukai number of a K3 surface, CRM Proc. and Lect. Notes 38 (2004), 177-192. MR2096145 (2005i:14046)

[8] D.R. Morrison, On K3 surfaces with large Picard number, Invent. Math. 75 (1984), 105-121. MR728142(85j:14071) 
[9] S. Mukai, Duality between $D(X)$ and $D(\widehat{X})$ with its applications to Picard sheaves, Nagoya Math. J. 81 (1981), 153-175. MR607081 (82f:14036)

[10] S. Mukai, On the moduli space of bundles on K3 surfaces, I, In: Vector Bundles on Algebraic Varieties, Oxford University Press, Bombay and London (1987), 341-413. MR893604 (88i:14036)

[11] S. Mukai, Duality of polarized K3 surfaces, New trends in algebraic geometry (Warwick, 1996), London Math. Soc. Lecture Note Ser. 264, Cambridge Univ. Press, Cambridge (1999), 311-326. MR.1714828 (2000i:14057)

[12] V.V. Nikulin, Integral symmetric bilinear forms and some of their applications, Math. USSR Izvestija 14 (1980), 103-167.

[13] V.V. Nikulin, Correspondences of a K3 surfaces with itself via moduli of sheaves. I, math.AG/0609233.

[14] K. Oguiso, K3 surfaces via almost-primes, Math. Research Letters 9 (2002), 47-63. MR.1892313 (2002m:14031)

[15] D. Orlov, Equivalences of derived categories and K3 surfaces, J. Math. Sci. 84 (1997), 13611381. MR1465519 (99a:14054)

[16] B. Saint-Donat, Projective models of K-3 surfaces, Amer. J. Math. 96 (1974), 602-639. MR0364263 (51:518)

[17] F. Scattone, On the compactification of moduli spaces for algebraic K3 surfaces, Mem. Amer. Math. Soc. 70 (1987), no. 374. MR.912636 (88m:14030)

[18] P. Stellari, Some remarks about the FM-partners of K3 surfaces with Picard numbers 1 and 2, Geom. Dedicata 108 (2004), 1-13. MR2112663 (2005i:14047)

[19] H. Sterk, Finiteness results for algebraic K3 surfaces, Math. Z. 189 (1985), 507-513. MR786280 (86j:14038)

[20] J. Wehler, K3 surfaces with Picard number 2, Arch. Math. 50 (1988), 73-82. MR925498 (89b:14054)

Dipartimento di Matematica "F. Enriques", Università degli Studi di Milano, Via Cesare Saldini 50, 20133 Milano, Italy

E-mail address: Paolo.Stellari@mat.unimi.it 